\title{
Generation and Dissemination of a National Virtual 3D City and Landscape Model for the Netherlands
}

\author{
Sander Oude Elberink, Jantien Stoter, Hugo Ledoux, and Tom Commandeur
}

\begin{abstract}
This paper describes the generation and dissemination of a national three-dimensional (3D) dataset representing the virtual and landscape model. The 3D model is produced automatically by fusing a two-dimensional (2D) national objectoriented database describing the physical landscape and the national high-resolution height model of the Netherlands. Semantic constraints are introduced to correctly model 3D objects. Three areas from different regions in the Netherlands have been processed in order to develop, improve, and test the automatic generation of a national 3D city and landscape model. Specific attention has been paid to exceptional cases that may occur in a nationwide dataset. Based on the test results, the Kadaster, the national agency in the Netherlands responsible for the production of nation wide geo-information, decided that it is feasible to produce a national 3D city and landscape model that fulfills the specifications that were defined as part of this study. Future research is identified to make the results further ready for practice.
\end{abstract}

\section{Introduction}

Over the past two years, a uniform approach for acquiring, storing, and visualizing 3D geo-information has been explored in a pilot in The Netherlands. In this pilot, over 65 private, public, and scientific organizations have collaborated to push the use of 3D information. The pilot project established the groundwork for a comprehensive national 3D geo-information program. A major result was a proof of concept for a 3D spatial data infrastructure (SDI) covering issues on the acquisition, standardization, storage, and use of 3D data (Stoter et al., 2011).

Besides the need for a national 3D standard realized as City Geography Markup Language (CITYGML) implementation (OGC, 2008 and 2012) (see Van den Brink et al., 2012), the pilot showed the need for a nationwide 3D city and landscape model. This model can serve as a reference for (new) 3D

Sander Oude Elberink is with the Faculty of Geo-Information Science and Earth Observation, University of Twente, Hengelosestraat 99, 7514 AE Enschede, The Netherlands (oudeelberink@itc.nl).

Jantien Stoter is with OTB, Delft University of Technology, Jaffalaan 9, 2600 AA, Delft, The Netherlands; Kadaster, P.O. Box 9046, 7300 GH Apeldoorn, The Netherlands; and Geonovum, Barchman Wuytierslaan 10, 3818 LH, Amersfoort, The Netherlands.

Hugo Ledoux is with OTB, Delft University of Technology, Jaffalaan 9, 2600 AA, Delft, The Netherlands.

Tom Commandeur is with HydroLogic Research BV, Elektronicaweg 2, 2628 XG, Delft, The Netherlands. information in a 3D virtual world, and as a basis for 3D planning and management of public space. The 3D base model can be further refined when a project develops. Many large municipalities have 3D data sets, but these are specifically required for the territory of the city and in various formats and resolutions. The pilot has shown promising results for generating a national 3D city and landscape model as a combination of 2D topography with high-resolution airborne Light Detection and Ranging (lidar) data. This paper describes how those results are further developed to generate a national 3D topographic dataset covering the whole of the Netherlands.

The term "topography" may be multi-interpretable, especially in combination of different types of dimensionalities: for some readers the term "topography" implies the relief description of the terrain, for others it means an object-based representation of elements from a cartographic map. The latter definition is the one that we use in this paper, and it refers to both natural features (rivers and forests) and man-made features (buildings and roads). In addition, three types of dimensionality of topographic objects are used: 2D, 2.5D, and 3D. In our context, topographic 2D data are represented as a cartographic vector map describing the objects such as roads, water, buildings, terrain, vegetation, engineering objects, etc. that we use as source data. 2.5D objects are part of the generated 3D data describing the surfaces in the landscape model (e.g., roads, terrain, water polygons; these can be projected to the $2 \mathrm{D}$ plane, but are geometrically represented by $2.5 \mathrm{D}$ height surfaces), whereas 3D objects refer to volumetric objects in the generated 3D data (mainly buildings). In the generated 3D data road junctions are built up from two 2.5D surfaces representing both the lower and upper road surface. In the remainder of this paper, the term "3D data" refers to the output of our algorithm (containing both 2.5D and 3D objects), whereas "2D data" refers to the input map data source.

This work is collaboration between the University of Twente, the Delft University of Technology, and the Kadaster, the national agency in the Netherlands responsible for the production and provision of nation wide geo-information. Three areas from different regions in the Netherlands have been processed in order to develop, improve and test the automatic generation of a 3D dataset. The test areas include complex interchanges, buildings above roads, bridges, urban areas, and forested areas.

Photogrammetric Engineering \& Remote Sensing Vol. 79, No. 2, February 2013, pp. 147-158.

0099-1112/13/7902-147/\$3.00/0

(C) 2013 American Society for Photogrammetry and Remote Sensing 